\title{
Drug release assays from new chitosan/pHEMA membranes obtained by gamma irradiation
}

\author{
M.H. Casimiro ${ }^{\text {a,* }}$, M.H. Gil ${ }^{\text {b }}$, J.P. Leal ${ }^{\mathrm{c}, \mathrm{d}}$ \\ ${ }^{a}$ Department of Physics, Nuclear and Technological Institute, E.N. 10, 2686-953 Sacavém, Portugal \\ ${ }^{\mathrm{b}}$ Department of Chemical Engineering, Faculty of Science and Technology, University of Coimbra, Pólo II Pinhal de Marrocos, \\ 3030-290 Coimbra, Portugal \\ ${ }^{\mathrm{c}}$ Department of Chemistry, Nuclear and Technological Institute, E.N. 10, 2686-953 Sacavém, Portugal \\ ${ }^{\mathrm{d}}$ Chemistry and Biochemistry Centre and Department of Chemistry and Biochemistry, Faculty of Sciences, \\ University of Lisboa, 1749-016 Lisboa, Portugal
}

Available online 8 September 2007

\begin{abstract}
With the purpose of obtaining a biocompatible and microbiologically safe matrix that simultaneously could be used as wound dressing material and as a controlled drug release system, membranes with different thickness and different contents in chitosan and hydroxyethyl methacrylate (HEMA) have been prepared by $\gamma$ irradiation from a ${ }^{60} \mathrm{Co}$ source. Antibiotic release experiments were performed before or after irradiation over amoxicillin loaded chitosan/pHEMA membranes in physiological saline solution, and monitored by UV-Vis spectrometry.

Results point out a fast amoxicillin release with similar release profile in all studied membranes. The amount of released drug was shown to be dependent on membranes network crosslinking due composition, radiation and membrane thickness.

(C) 2007 Elsevier B.V. All rights reserved.
\end{abstract}

PACS: $81.05 . \mathrm{Lg} ;$ 82.35.Jk; 82.35.Pq; 82.50.Kx

Keywords: Drug release; Gamma radiation, chitosan; Membranes; HEMA; Amoxicillin

\section{Introduction}

Most drugs can be administered by a variety of routes, broadly defined as local and systemic. Drugs administered systemically are absorbed into blood stream and distributed throughout the host patient via the circulatory system, which can result in microbiological resistance [1]. Sitespecific release systems limit the adverse effects of systemic administration and offer distinctive advantages over the classical methods of drug delivery. These include improvement of delivery efficacy, reduced toxicity and improved patient compliance and convenience $[1,2]$.

In the specific case of wound dressing, the possibility of simultaneous rapid and proper healing of the damage

\footnotetext{
* Corresponding author. Fax: +351 219941525.

E-mail address: casimiro@itn.pt (M.H. Casimiro).
}

tissues with local drug treatment would be an important advantage. Polysaccharides, like chitosan and its derivatives, have been considered to be advantageous in their application as a wound dressing material since they may, by themselves, actively participate in the process of wound healing [3-5].

Chitosan [poly- $\beta(1-4)-D-g l u c o s a m i n e]$ is a cationic polysaccharide of natural origin that is obtained by alkaline deacetylation of chitin, the principal exoskeleton component in crustaceans. Due to an unusual combination of properties such as non-toxicity, biocompatibility, biodegradability, bioactivity, acceleration of wound healing, fat biding capacity, etc., biomedical and environmental applications of chitosan and its chemically modified structures have been reported [2,3,6-10].

Among possible physical-chemical modifications need to adequate the polymer to each application, graft 
copolymerization induced by gamma radiation has revealed itself a very attractive technique since it could enable a wide variety of molecular designs to afford novel types of tailored hybrid materials [11-14].

In previous work $[11,12]$ we have evaluated the effect of various synthesis conditions on some physical, chemical and microbiological properties of chitosan/poly(hydroxyethyl methacrylate) copolymers obtained by $\gamma$ irradiation from a ${ }^{60} \mathrm{Co}$ source. pHEMA is a synthetic hydrogel which possesses high mechanical strength and biocompatibility [15]. Since natural macromolecular materials such as chitosan are usually mechanically weak and often difficult to process as membranes, the conjunction of these two components may result in a new matrix that combines the useful properties of both polymers. The purpose of this study has been to assess the possibilities of obtaining chitosan/pHEMA membranes that would maintain the wound dressing ability of chitosan backbone and simultaneously could be used as a biocompatible and microbiologically safe dermal matrix for controlled drug release. The drug in use was amoxicillin, which is a moderate-spectrum antibiotic (active against a wide range of gram-positive and a limited range of gram-negative organisms) widely used for the treatment of skin infections among others infections.

The work was divided in two parts and, here, only drug release experimental data are presented. In vitro haemolysis tests and extensive microbiological experiments have been submitted to appreciation for publication elsewhere.

\section{Experimental}

\subsection{Materials}

Chitosan medium molecular weight $\left(1.9 \times 10^{5}-3.1 \times\right.$ $\left.10^{5} \mathrm{Da}\right) 75-85 \%$ deacetylated was obtained from Aldrich Chemical Company, Inc., Milwaukee, USA. This was triturated $(\varphi<500 \mu \mathrm{m})$ and dried under vacuum at $313 \mathrm{~K}$. Hydroxyethyl methacrylate (HEMA), stabilised, 98\%, was obtained from ACROS Organics, Belgium, and used as received. All other chemical used were of analytical grade and used as received.

\subsection{Preparation of chitosan/pHEMA membranes}

The membranes preparation was achieved by mixing different chitosan solutions $(1 \%, 3 \%$ and $5 \% \mathrm{w} / \mathrm{V}$ of chitosan in acetic acid $1 \%)$ with HEMA monomer $(1 \%, 3 \%$ and $5 \% \mathrm{~V} / \mathrm{V}$ of the final mixture). The bubble-free aqueous solutions were poured on a clean glass plate in a dust-free atmosphere and allowed to dry at room temperature. The membranes thus formed were washed with $\mathrm{NaOH} 1 \%$ and then with distilled water and carefully peeled off from the glass plate.

Membranes were dried to constant weight in vacuum at $313 \mathrm{~K}$ and then immersed for $2 \mathrm{~h}$ in amoxicillin solution $(100 \mathrm{mg} / \mathrm{ml}$ in saline solution $(\mathrm{NaCl} 0.9 \% \mathrm{w} / \mathrm{V}))$. Dependent on membranes composition the thickness range of swollen samples varied within 50 and $420 \mu \mathrm{m}$.
Afterwards membranes were blotted free of surface drug solution with a filter paper, sealed in Amilon polyamide bags under nitrogen atmosphere and $\gamma$ irradiated at $0.6 \mathrm{kGy} \mathrm{h}^{-1}$ (4 kGy) using a ${ }^{60} \mathrm{Co}$ gamma source at room temperature.

The low dose rate and absorbed dose values which membranes were exposed to, were chosen based on the intrinsic behaviour of the system in study versus gamma radiation (chitosan is a degradative type polymer) and on previous membranes microbiological experiments (being naturally low contaminate, is expected that chitosan based membranes in study would need very low doses to assure the standard microbiological safety) [12]. Moreover, and in spite of other studies have reported the feasibility of gamma radiation $(25 \mathrm{kGy})$ as a sterilization method of amoxicillin [16], lower radiation doses would minimize eventual induced degradation products as well as adverse side effects on physical and chemical matrix properties $[11,17]$.

\subsection{Characterisation of chitosan/pHEMA membranes - Antibiotic release studies}

Antibiotic release experiments were divided in irradiated and non-irradiated groups and performed over amoxicillin loaded membranes with different contents of chitosan/ HEMA and different thickness.

Each membrane drug loaded was immersed in $100 \mathrm{ml}$ of physiological saline solution at $310 \mathrm{~K}$ with slight orbital stirring. The released amoxicillin was monitored at $276 \mathrm{~nm}$ on a UV-Vis-NIR spectrophotometer (Cary 5G, Varian, Switzerland), until no further changes of absorbance values were observed. These absorbance measurements were converted into amoxicillin concentrations using an adequate amoxicillin calibration curve and the total amount of released drug was calculated using three replicates for each membrane composition/thickness. To determine the amount of amoxicillin initially loaded, membranes were weight prior (in dried state) and after drug loaded procedure (in swollen state).

\section{Results and discussion}

In order to investigate the feasibility of using the prepared membranes as a matrix for controlled drug release, amoxicillin (as a model drug) was loaded into chitosan/ pHEMA.

Fig. 1 shows the release profiles in saline solution of amoxicillin from chitosan/pHEMA irradiated and nonirradiated membranes with different content in chitosan $(1 \%$ and $3 \%$ ) and HEMA $1 \%$ (mean \pm SD from three independent experiments). Swollen membranes presented a thickness of 150 and $180 \mu \mathrm{m}$ respectively for chitosan $1 \%$ and chitosan $3 \%$ content.

Results indicate that both formulations present similar release profiles (contrary to the initially expected), with an initial burst effect that may be attributed to the diffusion 


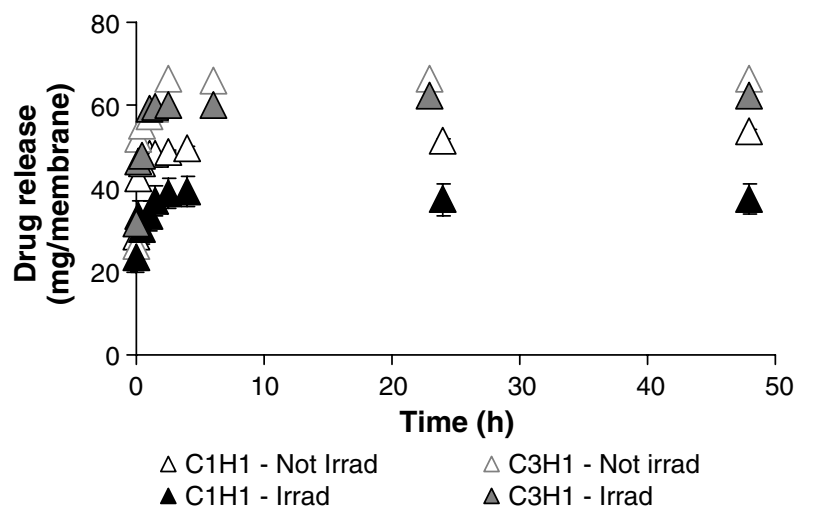

Fig. 1. Amoxicillin release profiles in saline solution at $310 \mathrm{~K}$ of nonirradiated and $\gamma$-irradiated chitosan/pHEMA membranes $\left(\mathrm{C}_{x} \mathrm{H}_{y-x}\right.$ indicates the percentage of chitosan and $y$ the percentage of HEMA).

of the drug and also to the release of adsorbed drug towards the surface matrix as reported by other authors with different polymeric matrix [18]. Furthermore, this drug release behaviour is also extended to all prepared membranes independently of composition and thickness. In this way, all formulations present a fast release of amoxicillin that result in a maximum availability of the drug in 1-2 $\mathrm{h}$, fact that may have to be with membranes formulation used (ratio of chitosan/pHEMA does not vary significantly in composition terms, only in membranes density).

From Fig. 1 is still possible to observe a slight drug release fall after $\gamma$ irradiation result of an increase in network crosslinking density (as suggested by previous scanning electron microscopy due to the reduction in porous size network [12]), and consequently a higher amount of entrapped drug.

Moreover, as represented membranes present similar thickness values it would be expected to tailor drug release rate by changing that feature. However it did not come to be. Results show that for each membrane composition studied, the convenient selection of membranes thickness allows us to tailor drug absorbed/released amount (instead of drug release rate) as can be seen in Fig. 2(a).

Fig. 2(a) exhibits the maximum drug absorption and drug release (in mg of drug per membrane) of all different $\gamma$-irradiated chitosan based membranes obtained. It suggests that maximum values of drug absorption/release are essentially dependent on membranes chitosan content given that chitosan 3\% based membranes presents very similar values when compared with chitosan $1 \%$ based membranes. It seems that an increase in membranes chitosan content leads to a shrinkage of the porous network when irradiated and consequently to the diminution of amoxicillin release efficiency after irradiation due drug entrapment as mentioned before.

At the same time it can be seen that enlarging membranes thickness enables higher drug absorption values (in non-irradiated membranes), but induces minor drug release amounts in irradiated ones due to the increasing
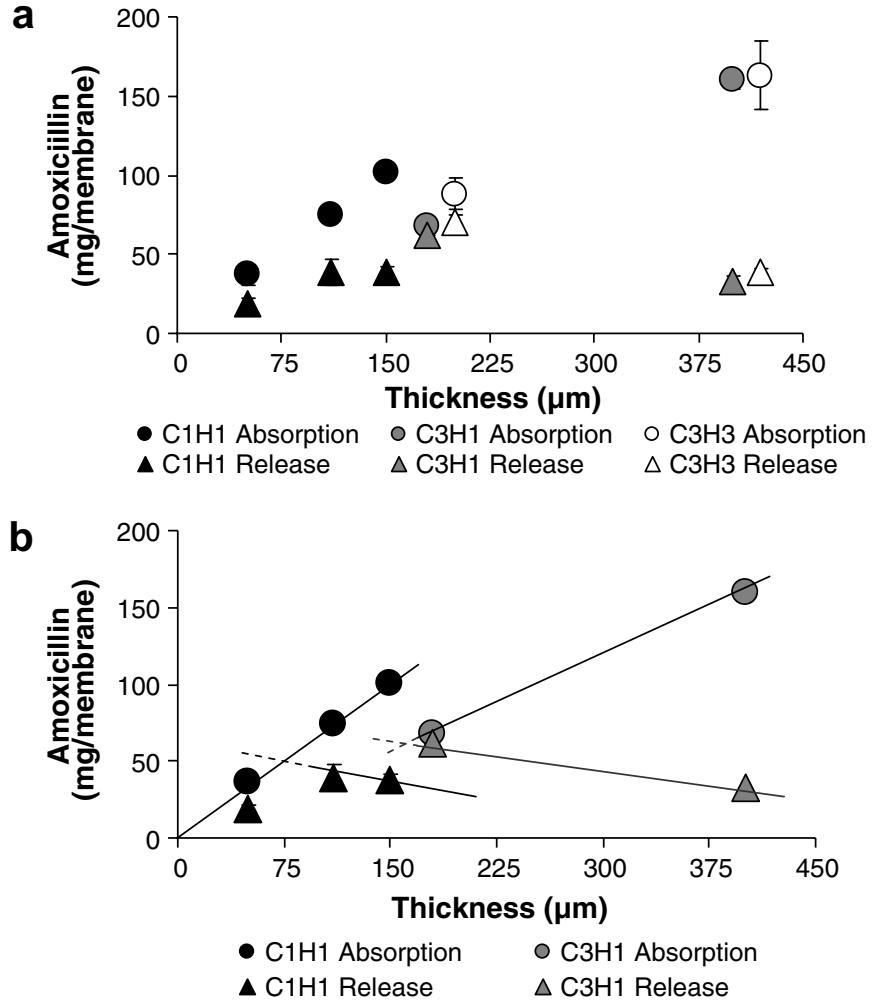

Fig. 2. (a) Drug absorption and drug release behaviour of different $\gamma$ irradiated chitosan based membranes. (b) Drug absorption and drug release behaviour of different $\gamma$-irradiated chitosan/pHEMA membranes Interception of curves tendency fitting.

network crosslinking density as earlier mentioned. Fig. 2(b) points out the behaviour just mentioned by exhibiting the curves tendency fitting of drug absorption/release displayed data.

Drug release data can also be expressed in percentage as shown in Fig. 3. Results let to anticipate an optimum membrane thickness range for amoxicillin release efficiency after irradiation. To determinate it, membranes composition, drug absorption and drug release curves ( $\mathrm{mg} / \mathrm{membrane})$ have to be considered.

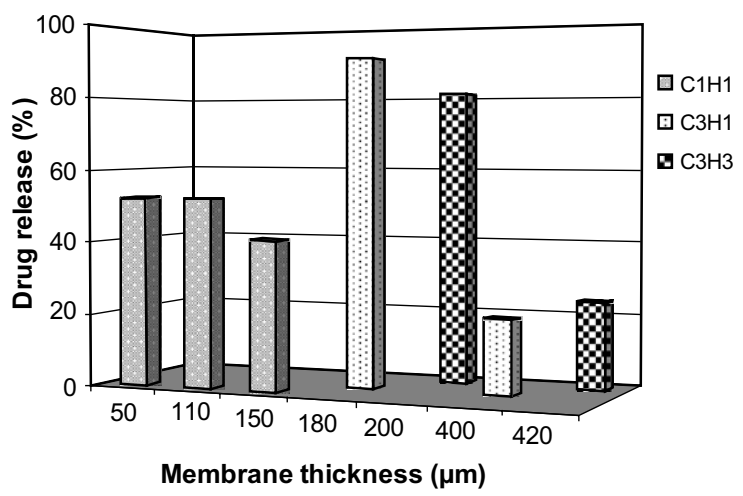

Fig. 3. Maximum amoxicillin released of $\gamma$-irradiated chitosan/pHEMA membranes. 


\section{Conclusions}

In the present study attention has been paid to the amoxicillin release kinetics of gamma irradiated chitosan/ pHEMA membranes. Results point out a fast amoxicillin release with similar release profile in all studied membranes contrary to the initially expected. According to obtained data it appears to be that the amount of released drug is dependent on membranes network crosslinking and in this way the amount of released drug can be tailored according to needs by selecting adequate composition and thickness. Obtained results also suggest the existence of an optimum membrane thickness range for amoxicillin release efficiency.

However, if only wound dressing features are being considered it might come out a different optimum membrane thickness, since the swelling properties are important to mimic the skin's moisture. As this study aim to obtain a matrix that simultaneously could be used as wound dressing material and as a controlled drug release system, particular attention should be done to membranes thickness once it can shape both features response.

\section{Acknowledgements}

This work has been supported by Operational Program of Science, Technology and Innovation from Third European Community Framework Program and from Foundation of Science and Technology, Portugal (grant SFRH/ $\mathrm{BD} / 2862 / 2000$ ).

\section{References}

[1] A.C. Queiroz, J.D. Santos, F.J. Monteiro, I.R. Gibson, J.C. Knowles, Biomaterials 22 (2001) 1393.

[2] M.V. Risbud, A.A. Hardikar, S.V. Bhat, R.R. Bhonde, J. Control. Release 68 (2000) 23.

[3] M. Ishihara, K. Nakanishi, K. Ono, M. Sato, M. Kikuchi, Y. Saito, H. Yura, T. Matsui, H. Hattori, M. Uenoyama, A. Kurita, Biomaterials 23 (2002) 833.

[4] C. Shi, Y. Zhu, X. Ran, M. Wang, Y. Su, T. Cheng, J. Surg. Res. 133 (2006) 185.

[5] C. Alemdaroglu, Z. Degin, N. Çelebi, F. Zor, S. Ozturk, D. Erdogan, Burns 32 (2006) 319

[6] D.V. Luyen, D.M. Huong, in: J.C. Salamone (Ed.), Polymeric Materials Encyclopaedia, Vol. 2, CRC Press, New York, 1996, p. 1208.

[7] S. Torrado, P. Prada, P.M. de la Torre, S. Torrado, Biomaterials 25 (2004) 917.

[8] S.V. Madihally, H.W.T. Mathew, Biomaterials 20 (1999) 1133.

[9] S.S. Koide, Nutri. Res. 18 (1998) 1091.

[10] R. Jayakumar, M. Prabaharan, R.L. Reis, J.F. Mano, Carbohyd. Polym. 62 (2005) 142.

[11] M.H. Casimiro, M.L. Botelho, J.P. Leal, M.H. Gil, Radiat. Phys. Chem. 72 (2005) 731.

[12] M.H. Casimiro, J.P. Leal, M.H. Gil, Nucl. Instr. and Meth. B 236 (2005) 482.

[13] L.M. Ferreira, A.N. Falcão, M.H. Gil, Mat. Sci. Forum 514-516 (2006) 1034

[14] S.R. Gomes, F.M.A. Margaça, I.M. Miranda Salvado, L.M. Ferreira, A.N. Falcão, Nucl. Instr. and Meth. B 248 (2006) 291.

[15] G. Bayramoglu, M.Y. Arica, Colloid Surf. A 202 (2002) 41.

[16] L. Valvo, L. Manna, R. Alimenti, S. Alimonti, P. Bertocchi, E. Ciranni, J. Pharm. Biomed. Anal. 21 (1999) 9.

[17] L.Y. Lim, E. Khor, O. Koo, J. Biomed. Res. (Appl. Biomater.) 43 (1998) 282.

[18] J.L. Whitby, Radiat. Phys. Chem. 42 (1993) 577. 